\title{
Existence, Uniqueness and Ergodicity of Positive Solution of Mutualism System with Stochastic Perturbation
}

\author{
Chunyan Ji, ${ }^{1,2}$ Daqing Jiang, ${ }^{2}$ Hong Liu, ${ }^{2,3}$ and Qingshan Yang ${ }^{2}$ \\ ${ }^{1}$ School of Mathematics and Statistics, Changshu Institute of Technology, Changshu, Jiangsu 215500, China \\ ${ }^{2}$ School of Mathematics and Statistics, Northeast Normal University, Changchun, Jilin 130024, China \\ ${ }^{3}$ China Economics and Management Academy, CIAS, Central University of Finance and Economics, \\ Beijing 100081, China
}

Correspondence should be addressed to Chunyan Ji, chunyanji80@hotmail.com

Received 30 December 2009; Accepted 15 June 2010

Academic Editor: Ben T. Nohara

Copyright (C) 2010 Chunyan Ji et al. This is an open access article distributed under the Creative Commons Attribution License, which permits unrestricted use, distribution, and reproduction in any medium, provided the original work is properly cited.

We discuss a two-species Lotka-Volterra mutualism system with stochastic perturbation. We show that there is a unique nonnegative solution of this system. Furthermore, we investigate that there exists a stationary distribution for this system, and it has ergodic property.

\section{Introduction}

It is well known that the differential equation

$$
\begin{aligned}
& \dot{x}_{1}(t)=x_{1}(t)\left[r_{1}-a_{11} x_{1}(t)+a_{12} x_{2}(t)\right], \\
& \dot{x}_{2}(t)=x_{2}(t)\left[r_{2}+a_{21} x_{1}(t)-a_{22} x_{2}(t)\right]
\end{aligned}
$$

denotes the population growth of mutualism system for the two species. $x_{1}(t)$ and $x_{2}(t)$ represent the densities of the two species at time $t$, respectively, and the parameters $r_{i}, a_{i j}, i, j=$ 1,2 are all positive. Goh [1] showed that the asymptotic stability equilibrium state of (1.1) in local must be asymptotic stability in global. That is, if $r_{i}>0, a_{i j}>0, i, j=1,2$, and $a_{11} a_{22}-a_{12} a_{21}>0$, then

$$
x_{1}(t) \longrightarrow x_{1}^{*}, \quad x_{2}(t) \longrightarrow x_{2}^{*}, \quad \text { as } t \longrightarrow \infty,
$$


where $x^{*}=\left(x_{1}^{*}, x_{2}^{*}\right)$ is the unique positive equilibrium of system (1.1) and

$$
x_{1}^{*}=\frac{r_{1} a_{22}+r_{2} a_{12}}{a_{11} a_{22}-a_{12} a_{21}}>0, \quad x_{2}^{*}=\frac{r_{2} a_{11}+r_{1} a_{21}}{a_{11} a_{22}-a_{12} a_{21}}>0 .
$$

While if $a_{11} a_{22}-a_{12} a_{21}<0$, then the population of both species increase to infinite. There are extensive literature concerned with mutualism system; see [2-7].

The papers mentioned above are all deterministic models, which do not incorporate the effect of fluctuating environment. In fact, environmental fluctuations are important components in the population system. Most of natural phenomena do not follow strictly deterministic laws, but rather oscillate randomly around some average values, hence the deterministic equilibrium is no longer an absolutely fixed state [8]. Therefore stochastic differential equation models play a significant role in various branches of applied sciences including the population system, as they provide some additional degree of realism compared to their deterministic counterpart [9-13]. Recently, many authors have paid attention to how population systems are affected by random fluctuations from environment (see, e.g., [14-18]). However, as far as we known, there is few paper consider how environmental noises affect the dynamical behaviors of the mutualism system, Zeng et al. [19] discussed the effects of noise and time delay on $C(s)$ (the normalized correlation function) and $T_{c}$ (the associated relaxation time) of a mutualism system, in which they considered the intraspecies interaction parameters were stochastically perturbed. Motivated by this, the main aim of this paper is to study the dynamical behaviors of the mutualism system with stochastic perturbation.

In this paper, considering the effect of randomly fluctuating environment, we incorporate white noise in each equation of system (1.1). Here we assume that fluctuations in the environment will manifest themselves mainly as fluctuations in the natural growth rates $r_{i}, i=1,2$. Suppose $r_{i} \rightarrow r_{i}+\sigma_{i} \dot{B}_{i}(t)$, where $B_{i}(t), i=1,2$ are mutually independent one dimensional standard Brownian motions with $B_{i}(0)=0$, and $\sigma_{i}>0, i=1,2$ are the intensities of white noises. The stochastic version corresponding to the deterministic system (1.1) takes the following form:

$$
\begin{aligned}
& d x_{1}(t)=x_{1}(t)\left[\left(r_{1}-a_{11} x_{1}(t)+a_{12} x_{2}(t)\right) d t+\sigma_{1} d B_{1}(t)\right] \\
& d x_{2}(t)=x_{2}(t)\left[\left(r_{2}+a_{21} x_{1}(t)-a_{22} x_{2}(t)\right) d t+\sigma_{2} d B_{2}(t)\right] .
\end{aligned}
$$

This paper is organized as follows. In Section 2, we show there is a unique positive solution of (1.4) if $a_{11} a_{22}-a_{12} a_{21}>0$, and give out the estimation of the solution. The stability of system (1.4) is investigated in Section 3. Since (1.4) does not have interior equilibrium, we cannot discuss the stability as the deterministic system. First, we show there is a stationary distribution of (1.4) and it has ergodic property. Next, by estimating the $p$ moment, we explore some properties of the solution.

Throughout this paper, unless otherwise specified, let $\left(\Omega,\left\{\mathscr{F}_{t}\right\}_{t \geq 0}, P\right)$ be a complete probability space with a filtration $\left\{\mathcal{F}_{t}\right\}_{t \geq 0}$ satisfying the usual conditions (i.e., it is right continuous and $\mathcal{F}_{0}$ contains all $P$-null sets). Let $R_{+}^{2}$ denote the positive cone of $R^{2}$, namely $R_{+}^{2}=\left\{x \in R^{2}: x_{i}>0, i=1,2\right\}$. If $A$ is a vector, its transpose is denoted by $A^{\top}$. For $x \in R^{2},|x|=\left|x_{1}\right|+\left|x_{2}\right|$. 


\section{The Existence and Estimation of the Solution}

To investigate the dynamical behavior, the first concern thing is whether the solution is global existence. Moreover, for a population model, whether the value is nonnegative is also considered. Hence in this section we first show the solution of (1.4) is global and nonnegative. As we have known, for a stochastic differential equation to have a unique global solution for any given initial value, the coefficients of the equation are generally required to satisfy the linear growth condition and local Lipschitz condition (cf. Arnold [20], Mao [21]). However, the coefficients of (1.4) do not satisfy the linear growth condition, though they are locally Lipschitz continuous, so the solution of (1.4) may explode at a finite time. In this section, following the way developed by Mao et al. [17], we show there is a unique positive solution of (1.4).

Theorem 2.1. There is a unique positive solution $x(t)$ of system (1.4) for any given initial value $x(0)=x_{0} \in R_{+}^{2}$ provided $a_{11} a_{22}>a_{12} a_{21}$.

Proof. The proof is similar to Theorem 2.1 in [17]. Here we define a $C^{2}$-function $V: R_{+}^{2} \rightarrow R_{+}$:

$$
V\left(x_{1}, x_{2}\right)=a_{21}\left[x_{1}-x_{1}^{*}-x_{1}^{*} \log \frac{x_{1}}{x_{1}^{*}}\right]+a_{12}\left[x_{2}-x_{2}^{*}-x_{2}^{*} \log \frac{x_{2}}{x_{2}^{*}}\right]
$$

where $x^{*}=\left(x_{1}^{*}, x_{2}^{*}\right)^{\top}$ satisfies

$$
\begin{aligned}
& r_{1}-a_{11} x_{1}^{*}+a_{12} x_{2}^{*}=0, \\
& r_{2}+a_{21} x_{1}^{*}-a_{22} x_{2}^{*}=0 .
\end{aligned}
$$

Remark 2.2. Theorem 2.1 shows stochastic equation (1.4) also has a global positive solution under the same condition of the corresponding deterministic system (1.1). That is to say, the white noise does not affect the existence of the unique global positive solution.

In the remaining of this section, we give the estimation of the solution of system (1.4). Jiang and Shi [22] discussed a randomized nonautonomous logistic equation:

$$
d N(t)=N(t)[(a(t)-b(t) N(t)) d t+\alpha(t) d B(t)],
$$

where $B(t)$ is 1-dimensional standard Brownian motion, $N(0)=N_{0}$ and $N_{0}$ is independent of $B(t)$. They showed the following.

Lemma 2.3 (see [22]). Assume that $a(t), b(t)$ and $\alpha(t)$ are bounded continuous functions defined on $[0, \infty), a(t)>0$ and $b(t)>0$. Then there exists a unique continuous positive solution of (2.3) for any initial value $N(0)=N_{0}>0$, which is global and represented by

$$
N(t)=\frac{e^{\int_{0}^{t}\left[a(s)-\alpha^{2}(s) / 2\right] d s+\alpha(s) d B(s)}}{1 / N_{0}+\int_{0}^{t} b(s) e^{\int_{0}^{s}\left[a(\tau)-\alpha^{2}(\tau) / 2\right] d \tau+\alpha(\tau) d B(\tau)} d s}, \quad t \geq 0 .
$$


Theorem 2.4. Assume that $a_{11} a_{22}>a_{12} a_{21}$ and $x(t) \in R_{+}^{2}$ is the solution of system (1.4) with initial value $x_{0} \in R_{+}^{2}$. Then $x(t)$ has the property that

$$
x_{1}(t) \geq \phi_{1}(t), \quad x_{2}(t) \geq \phi_{2}(t),
$$

where $\phi_{1}(t)$ and $\phi_{2}(t)$ are the solutions of equations:

$$
\begin{array}{ll}
d \phi_{1}(t)=\phi_{1}(t)\left[\left(r_{1}-a_{11} \phi_{1}(t)\right) d t+\sigma_{1} d B_{1}(t)\right], & \phi_{1}(0)=x_{1}(0), \\
d \phi_{2}(t)=\phi_{2}(t)\left[\left(r_{2}-a_{22} \phi_{2}(t)\right) d t+\sigma_{2} d B_{2}(t)\right], & \phi_{2}(0)=x_{2}(0) .
\end{array}
$$

The result of Theorem 2.4 follows directly from the classical comparison theorem of stochastic differential equations (see [23]).

Remark 2.5. From Lemma 2.3, we see

$$
\phi_{1}(t)=\frac{e^{\left(r_{1}-\sigma_{1}^{2} / 2\right) t+\sigma_{1} B_{1}(t)}}{1 / x_{1}(0)+a_{11} \int_{0}^{t} e^{\left(r_{1}-\sigma_{1}^{2} / 2\right) s+\sigma_{1} B_{1}(s)} d s}, \quad \phi_{2}(t)=\frac{e^{\left(r_{2}-\sigma_{2}^{2} / 2\right) t+\sigma_{2} B_{2}(t)}}{1 / x_{2}(0)+a_{22} \int_{0}^{t} e^{\left(r_{2}-\sigma_{2}^{2} / 2\right) s+\sigma_{2} B_{2}(s)} d s} .
$$

This together with Theorem 2.4 shows that if $r_{i}>\left(\sigma_{i}^{2} / 2\right)(i=1,2)$, then both species will not extinct.

\section{Stationary Distribution and Ergodicity for System (1.4)}

In the introduction, we have mentioned that if $r_{i}>0, a_{i j}>0, i, j=1,2$, and $a_{11} a_{22}-a_{12} a_{21}>$ 0 , then the unique positive equilibrium $\left(x_{1}^{*}, x_{2}^{*}\right)$ of $(1.1)$ is globally stable. But there is none positive equilibrium for (1.4). We investigate there is a stationary distribution for system (1.4) instead of asymptotically stable equilibria [24]. Before giving the main theorem, we first give a lemma (see [25]).

Assumption $B$. There exists a bounded domain $U \subset E_{l}$ with regular boundary $\Gamma$, having the following properties.

(B.1) In the domain $U$ and some neighbourhood thereof, the smallest eigenvalue of the diffusion matrix $A(x)$ is bounded away from zero.

(B.2) If $x \in E_{l} \backslash U$, the mean time $\tau$ at which a path issuing from $x$ reaches the set $U$ is finite, and $\sup _{x \in K} E_{x} \tau<\infty$ for every compact subset $K \subset E_{l}$.

Lemma 3.1 (see [25]). If $(B)$ holds, then the Markov process $X(t)$ has a stationary distribution $\mu(A)$. Let $f(\cdot)$ be a function integrable with respect to the measure $\mu$. Then

$$
P_{x}\left\{\lim _{T \rightarrow \infty} \frac{1}{T} \int_{0}^{T} f(X(t)) d t=\int_{E_{l}} f(x) \mu(d x)\right\}=1
$$

for all $x \in E_{l}$. 
Theorem 3.2. Assume $a_{11} a_{22}>a_{12} a_{21}, \sigma_{1}>0, \sigma_{2}>0$ and $\delta<\min \left\{m_{1}\left(x_{1}^{*}\right)^{2}, m_{2}\left(x_{2}^{*}\right)^{2}\right\}$. Then there is a stationary distribution $\mu(A)$ for system (1.4) and it has ergodic property. Here $\left(x_{1}^{*}, x_{2}^{*}\right)$ is the solution of (2.2), $\delta=\left(a_{21} x_{1}^{*} \sigma_{1}^{2}+a_{12} x_{2}^{*} \sigma_{2}^{2}\right) / 2, m_{1}=a_{11} a_{21}-a_{12} a_{21} / \epsilon_{0}>0$ and $m_{2}=$ $a_{12} a_{22}-\epsilon_{0} a_{12} a_{21}>0$, where $\epsilon_{0}>0$ satisfies $a_{12} / a_{11}<\epsilon_{0}<a_{22} / a_{21}$.

Proof. Define $V: E_{l}=R_{+}^{2} \rightarrow R_{+}$:

$$
V\left(x_{1}, x_{2}\right)=a_{21}\left(x_{1}-x_{1}^{*}-x_{1}^{*} \log \frac{x_{1}}{x_{1}^{*}}\right)+a_{12}\left(x_{2}-x_{2}^{*}-x_{2}^{*} \log \frac{x_{2}}{x_{2}^{*}}\right) .
$$

Then

$$
\begin{aligned}
d V= & a_{21}\left(1-\frac{x_{1}^{*}}{x_{1}}\right) d x_{1}+\frac{a_{21}}{2} \frac{x_{1}^{*}}{x_{1}^{2}}\left(d x_{1}\right)^{2}+a_{12}\left(1-\frac{x_{2}^{*}}{x_{2}}\right) d x_{2}+\frac{a_{12}}{2} \frac{x_{2}^{*}}{x_{2}^{2}}\left(d x_{2}\right)^{2} \\
= & a_{21}\left(x_{1}-x_{1}^{*}\right)\left[\left(r_{1}-a_{11} x_{1}+a_{12} x_{2}\right) d t+\sigma_{1} d B_{1}(t)\right]+\frac{1}{2} a_{21} x_{1}^{*} \sigma_{1}^{2} d t \\
& +a_{12}\left(x_{2}-x_{2}^{*}\right)\left[\left(r_{2}+a_{21} x_{1}-a_{22} x_{2}\right) d t+\sigma_{2} d B_{2}(t)\right]+\frac{1}{2} a_{12} x_{2}^{*} \sigma_{2}^{2} d t \\
:= & L V d t+a_{21} \sigma_{1}\left(x_{1}-x_{1}^{*}\right) d B_{1}(t)+a_{12} \sigma_{2}\left(x_{2}-x_{2}^{*}\right) d B_{2}(t),
\end{aligned}
$$

where

$$
\begin{aligned}
L V= & a_{21}\left(x_{1}-x_{1}^{*}\right)\left(r_{1}-a_{11} x_{1}+a_{12} x_{2}\right)+\frac{1}{2} a_{21} x_{1}^{*} \sigma_{1}^{2} \\
& +a_{12}\left(x_{2}-x_{2}^{*}\right)\left(r_{2}+a_{21} x_{1}-a_{22} x_{2}\right)+\frac{1}{2} a_{12} x_{2}^{*} \sigma_{2}^{2} \\
= & a_{21}\left(x_{1}-x_{1}^{*}\right)\left[-a_{11}\left(x_{1}-x_{1}^{*}\right)+a_{12}\left(x_{2}-x_{2}^{*}\right)\right] \\
& +a_{12}\left(x_{2}-x_{2}^{*}\right)\left[a_{21}\left(x_{1}-x_{1}^{*}\right)-a_{22}\left(x_{2}-x_{2}^{*}\right)\right]+\delta \\
= & -a_{11} a_{21}\left(x_{1}-x_{1}^{*}\right)^{2}+2 a_{12} a_{21}\left(x_{1}-x_{1}^{*}\right)\left(x_{2}-x_{2}^{*}\right) \\
& -a_{12} a_{22}\left(x_{2}-x_{2}^{*}\right)^{2}+\delta,
\end{aligned}
$$

according to the equality (2.2). By Young inequality, we have

$$
2 a_{12} a_{21}\left|x_{1}-x_{1}^{*}\right|\left|x_{2}-x_{2}^{*}\right| \leq a_{12} a_{21}\left[\frac{\left(x_{1}-x_{1}^{*}\right)^{2}}{\epsilon_{0}}+\epsilon_{0}\left(x_{2}-x_{2}^{*}\right)^{2}\right] \text {. }
$$

Then

$$
\begin{aligned}
L V & \leq-\left(a_{11} a_{21}-\frac{a_{12} a_{21}}{\epsilon_{0}}\right)\left(x_{1}-x_{1}^{*}\right)^{2}-\left(a_{12} a_{22}-\epsilon_{0} a_{12} a_{21}\right)\left(x_{2}-x_{2}^{*}\right)^{2}+\delta \\
& =-m_{1}\left(x_{1}-x_{1}^{*}\right)^{2}-m_{2}\left(x_{2}-x_{2}^{*}\right)^{2}+\delta .
\end{aligned}
$$


Note that $\delta<\min \left\{m_{1}\left(x_{1}^{*}\right)^{2}, m_{2}\left(x_{2}^{*}\right)^{2}\right\}$; then the ellipsoid

$$
-m_{1}\left(x_{1}-x_{1}^{*}\right)^{2}-m_{2}\left(x_{2}-x_{2}^{*}\right)^{2}+\delta=0
$$

lies entirely in $R_{+}^{2}$. We can take $U$ to be any neighborhood of the ellipsoid with $\bar{U} \subseteq E_{l}=R_{+}^{2}$, so for $x \in U \backslash E_{l}, L V \leq 0$, which implies that condition (B.2) in Lemma 3.1 is satisfied. Besides, there is $M>0$ such that

$$
\sum_{i, j=1}^{n}\left(\sum_{k=1}^{n} g_{i k}(x) g_{j k}(x)\right) \xi_{i} \xi_{j}=\sigma_{1}^{2} x_{1}^{2} \xi_{1}^{2}+\sigma_{2}^{2} x_{2}^{2} \xi_{2}^{2} \geq M\left|\xi^{2}\right| \quad x \in \bar{U}, \xi \in R^{2},
$$

which implies condition (B.1) is also satisfied. Therefore, the stochastic system (1.4) has a stable a stationary distribution $\mu(A)$ and it is ergodic.

Remark 3.3. If $a_{11} a_{22}>a_{12} a_{21}$ and $a_{22}>a_{21}$, we can choose $\epsilon_{0}=(1 / 2)\left(a_{12} / a_{11}+a_{22} / a_{21}\right)$, then $m_{1}=a_{11} a_{12}\left(a_{22}-a_{21}\right) /\left(a_{11} a_{22}+a_{12} a_{21}\right)$ and $m_{2}=a_{12}\left(a_{11} a_{22}-a_{12} a_{21}\right) / 2 a_{11}$.

Since system (1.4) is ergodic, next we explore some properties of the solution.

Consider the equation

$$
\dot{N}(t)=N(t)[a-b N(t)]
$$

with initial value $N_{0}>0$. It is well known, when $a, b>0$, (3.9) has a unique positive solution

$$
\begin{gathered}
N(t)=\frac{e^{a t}}{1 / \widetilde{N}_{0}+(b / a)\left(e^{a t}-1\right)}, \quad t \geq 0, \\
\lim _{t \rightarrow \infty} N(t)=\frac{a}{b^{\prime}}, \quad \lim _{t \rightarrow \infty} \frac{\log N(t)}{t}=0 .
\end{gathered}
$$

Lemma 3.4. Suppose that $r_{1}>\sigma_{1}^{2} / 2$ and $\phi_{1}(t)$ is the solution of (2.6), then one has

$$
\psi_{1}(t) e^{-\sigma_{1}\left(\max _{0 \leq s \leq t} B_{1}(s)-B_{1}(t)\right)} \leq \phi_{1}(t) \leq \psi_{1}(t) e^{-\sigma_{1}\left(\min _{0 \leq s \leq t} B_{1}(s)-B_{1}(t)\right)}
$$

where $\psi_{1}(t)$ is the solution of

$$
\begin{gathered}
\dot{\psi}_{1}(t)=\psi_{1}(t)\left[r_{1}-\frac{\sigma_{1}^{2}}{2}-a_{11} \psi_{1}(t)\right], \\
\psi_{1}(0)=x_{1}(0) .
\end{gathered}
$$


Proof. From the representation of the solution $\phi_{1}(t)$, we have

$$
\begin{aligned}
\frac{1}{\phi_{1}(t)} & =\frac{1}{x_{1}(0)} e^{-\left(r_{1}-\sigma_{1}^{2} / 2\right) t-\sigma_{1} B_{1}(t)}+a_{11} \int_{0}^{t} e^{-\left(r_{1}-\sigma_{1}^{2} / 2\right)(t-s)-\sigma\left(B_{1}(t)-B_{1}(s)\right)} d s \\
& =e^{-\sigma_{1} B_{1}(t)}\left[\frac{1}{x_{1}(0)} e^{-\left(r_{1}-\sigma_{1}^{2} / 2\right) t}+a_{11} \int_{0}^{t} e^{-\left(r_{1}-\sigma_{1}^{2} / 2\right)(t-s)} e^{\sigma_{1} B_{1}(s)} d s\right] \\
& \leq e^{-\sigma_{1} B_{1}(t)}\left[\frac{1}{x_{1}(0)} e^{-\left(r_{1}-\sigma^{2} / 2\right) t}+a_{11} e^{\sigma_{1} \max _{0 \leq s \leq t} B_{1}(s)} \int_{0}^{t} e^{-\left(r_{1}-\sigma_{1}^{2} / 2\right)(t-s)} d s\right] \\
& \leq e^{\sigma_{1}\left[\max _{0 \leq s \leq t} B_{1}(s)-B_{1}(t)\right]}\left[\frac{1}{x_{1}(0)} e^{-\left(r_{1}-\sigma_{1}^{2} / 2\right) t}+a_{11} \int_{0}^{t} e^{-\left(r_{1}-\sigma_{1}^{2} / 2\right)(t-s)} d s\right],
\end{aligned}
$$

where the last inequality is based on the property of Brownian motion that $B(0)=0$. Similarly, we have

$$
\frac{1}{\phi_{1}(t)} \geq e^{\sigma_{1}\left(\min _{0 \leq s \leq t} B_{1}(s)-B_{1}(t)\right)}\left[\frac{1}{x_{1}(0)} e^{-\left(r_{1}-\sigma_{1}^{2} / 2\right) t}+a_{11} \int_{0}^{t} e^{-\left(r_{1}-\sigma_{1}^{2} / 2\right)(t-s)} d s\right] .
$$

Therefore

$$
e^{\sigma_{1}\left(\min _{0 \leq s \leq t} B_{1}(s)-B_{1}(t)\right)} \frac{1}{\psi_{1}(t)} \leq \frac{1}{\phi_{1}(t)} \leq e^{\sigma_{1}\left(\max _{0 \leq s \leq t} B_{1}(s)-B_{1}(t)\right)} \frac{1}{\psi_{1}(t)}
$$

which is as required.

Lemma 3.5. Suppose that $r_{1}>\sigma_{1}^{2} / 2$ and $\phi_{1}(t)$ is the solution of (2.6), then one has

$$
\lim _{t \rightarrow \infty} \frac{\log \phi_{1}(t)}{t}=0
$$

Proof. It is easy to drive from Lemma 3.4 that

$$
\sigma_{1}\left(B_{1}(t)-\max _{0 \leq s \leq t} B_{1}(s)\right) \leq \log \phi_{1}(t)-\log \psi_{1}(t) \leq \sigma_{1}\left(B_{1}(t)-\min _{0 \leq s \leq t} B_{1}(s)\right)
$$

Note that the distribution of $\max _{0 \leq s \leq t} B_{1}(s)$ is the same as $\left|B_{1}(t)\right|$, and that $\min _{0 \leq s \leq t} B_{1}(s)$ has the same distribution as $-\max _{0 \leq s \leq t} B_{1}(s)$, then by (3.11) and the strong law of large numbers, we get

$$
\lim _{t \rightarrow \infty} \frac{\log \phi_{1}(t)}{t}=\lim _{t \rightarrow \infty} \frac{\log \psi_{1}(t)}{t}=0
$$

This completes the proof of Lemma 3.5. 
Now consider the solution of (2.7), by the same reasons as Lemmas 3.4 and 3.5, we have the following.

Lemma 3.6. Suppose that $r_{2}>\sigma_{2}^{2} / 2$ and $\phi_{2}(t)$ is the solution of (2.7), then one has

$$
\lim _{t \rightarrow \infty} \frac{\log \phi_{2}(t)}{t}=0
$$

Lemma 3.7. Let $M(t)=\int_{0}^{t} e^{s} d B(s)$, where $B(t)$ is 1-dimensional standard Brownian motion, then

$$
\limsup _{t \rightarrow \infty} \frac{\left|e^{-t} M(t)\right|}{\sqrt{\log t}}=1 \quad \text { a.s. }
$$

Proof. The proof can be found on [21, page 70].

Based on these lemmas, now we show the main result in this section.

Let $y_{1}(t)=\log x_{1}(t), y_{2}(t)=\log x_{2}(t)$; then by Itô's formula we obtain

$$
\begin{aligned}
& d y_{1}(t)=\left(r_{1}-\frac{\sigma_{1}^{2}}{2}-a_{11} x_{1}(t)+a_{12} x_{2}(t)\right) d t+\sigma_{1} d B_{1}(t) \\
& d y_{2}(t)=\left(r_{2}-\frac{\sigma_{2}^{2}}{2}+a_{21} x_{1}(t)-a_{22} x_{2}(t)\right) d t+\sigma_{2} d B_{2}(t)
\end{aligned}
$$

If $a_{11} a_{22}>a_{12} a_{21}$ and $2 r_{1}>\sigma_{1}^{2}, 2 r_{2}>\sigma_{2}^{2}$, then the equation

$$
\begin{aligned}
& r_{1}-\frac{\sigma_{1}^{2}}{2}-a_{11} x_{1}+a_{12} x_{2}=0 \\
& r_{2}-\frac{\sigma_{2}^{2}}{2}+a_{21} x_{1}-a_{22} x_{2}=0
\end{aligned}
$$

has a unique positive solution:

$$
\tilde{x}_{1}^{*}=\frac{a_{22}\left(r_{1}-\sigma_{1}^{2} / 2\right)+a_{12}\left(r_{2}-\sigma_{2}^{2} / 2\right)}{a_{11} a_{22}-a_{12} a_{21}}, \quad \tilde{x}_{2}^{*}=\frac{a_{21}\left(r_{1}-\sigma_{1}^{2} / 2\right)+a_{11}\left(r_{2}-\sigma_{2}^{2} / 2\right)}{a_{11} a_{22}-a_{12} a_{21}}
$$


Lemma 3.8. Assume $a_{11} a_{22}>a_{12} a_{21}$ and $2 r_{1}>\sigma_{1}^{2}, 2 r_{2}>\sigma_{2}^{2}$. Then for any initial value $x_{0} \in R_{+}^{2}$, the solution $x(t)$ of system (1.4) has the following property:

$$
\lim _{t \rightarrow \infty} \bar{x}_{1}(t)=\tilde{x}_{1}^{*}, \quad \lim _{t \rightarrow \infty} \bar{x}_{2}(t)=\tilde{x}_{2}^{*}
$$

where $\bar{x}_{i}(t)=(1 / t) \int_{0}^{t} x_{i}(s) d s, i=1,2$.

Proof. It follows from (3.22) that

$$
\begin{aligned}
& \frac{y_{1}(t)}{t}=\frac{y_{1}(0)}{t}+r_{1}-\frac{\sigma_{1}^{2}}{2}-a_{11} \bar{x}_{1}(t)+a_{12} \bar{x}_{2}(t)+\sigma_{1} \frac{B_{1}(t)}{t}, \\
& \frac{y_{2}(t)}{t}=\frac{y_{2}(0)}{t}+r_{2}-\frac{\sigma_{2}^{2}}{2}+a_{21} \bar{x}_{1}(t)-a_{22} \bar{x}_{2}(t)+\sigma_{2} \frac{B_{2}(t)}{t} .
\end{aligned}
$$

Obviously, to prove the result, it is an easy consequence of

$$
\lim _{t \rightarrow \infty} \frac{y_{i}(t)}{t}=\lim _{t \rightarrow \infty} \frac{\log x_{i}(t)}{t}=0, \quad i=1,2 \text { a.s. }
$$

We first show that

$$
\liminf _{t \rightarrow \infty} \frac{\log x_{i}(t)}{t} \geq 0, \quad i=1,2 \text { a.s. }
$$

In fact, the results of Theorem 2.4 and Lemmas 3.5 and 3.6 imply that (3.28) is true.

Next, we will prove

$$
\limsup _{t \rightarrow \infty} \frac{\log x_{i}(t)}{t} \leq 0, \quad i=1,2 \text { a.s. }
$$

If $a_{11} a_{22}>a_{12} a_{21}$, then there exist positive constants $c_{1}, c_{2}, m_{1}, m_{2}$ such that

$$
\begin{gathered}
-a_{11} c_{1}+a_{21} c_{2}=-m_{1}, \\
a_{12} c_{1}-a_{22} c_{2}=-m_{2} .
\end{gathered}
$$


From (3.22) we get

$$
\begin{aligned}
d\left(c_{1} y_{1}(t)+c_{2} y_{2}(t)\right) & \\
= & c_{1} d \log x_{1}(t)+c_{2} d \log x_{2}(t) \\
= & c_{1}\left[\left(r_{1}-\frac{\sigma_{1}^{2}}{2}-a_{11} x_{1}(t)+a_{12} x_{2}(t)\right) d t+\sigma_{1} d B_{1}(t)\right] \\
& +c_{2}\left[\left(r_{2}-\frac{\sigma_{2}^{2}}{2}+a_{21} x_{1}(t)-a_{22} x_{2}(t)\right) d t+\sigma_{2} d B_{2}(t)\right] \\
= & {\left[\left(r_{1}-\frac{\sigma_{1}^{2}}{2}\right) c_{1}+\left(r_{2}-\frac{\sigma_{2}^{2}}{2}\right) c_{2}+\left(a_{21} c_{2}-a_{11} c_{1}\right) x_{1}(t)+\left(a_{12} c_{1}-a_{22} c_{2}\right) x_{2}(t)\right] d t } \\
& +c_{1} \sigma_{1} d B_{1}(t)+c_{2} \sigma_{2} d B_{2}(t) \\
= & {\left[\left(r_{1}-\frac{\sigma_{1}^{2}}{2}\right) c_{1}+\left(r_{2}-\frac{\sigma_{2}^{2}}{2}\right) c_{2}-m_{1} x_{1}(t)-m_{2} x_{2}(t)\right] d t } \\
& +c_{1} \sigma_{1} d B_{1}(t)+c_{2} \sigma_{2} d B_{2}(t), \\
d\left[e^{t}\left(c_{1} y_{1}(t)+c_{2} y_{2}(t)\right)\right] & +e^{t}\left[c_{1} \sigma_{1} d B_{1}(t)+c_{2} \sigma_{2} d B_{2}(t)\right] . \\
= & e^{t} d\left(c_{1} y_{1}(t)+c_{2} y_{2}(t)\right)+e^{t}\left(c_{1} y_{1}(t)+c_{2} y_{2}(t)\right) d t \\
= & e^{t}\left[\left(r_{1}-\frac{\sigma_{1}^{2}}{2}\right) c_{1}+\left(r_{2}-\frac{\sigma_{2}^{2}}{2}\right) c_{2}+c_{1} y_{1}(t)-m_{1} e^{y_{1}(t)}+c_{2} y_{2}(t)-m_{2} e^{y_{2}(t)}\right] d t \\
& {[t}
\end{aligned}
$$

Note that the function $c_{1} y_{1}-m_{1} e^{y_{1}}$ has its maximum value $c_{1}^{*}=c_{1} \log \left(c_{1} / m_{1}\right)-c_{1}$ at $y_{1}=$ $\log \left(c_{1} / m_{1}\right)$, and the function $c_{2} y_{2}-m_{2} e^{y_{2}}$ has its maximum value $c_{2}^{*}=c_{2} \log \left(c_{2} / m_{2}\right)-c_{2}$ at $y_{2}=\log \left(c_{2} / m_{2}\right)$; then

$$
\begin{aligned}
d\left[e^{t}\left(c_{1} y_{1}(t)+c_{2} y_{2}(t)\right)\right] \leq & e^{t}\left[\left(r_{1}-\frac{\sigma_{1}^{2}}{2}\right) c_{1}+\left(r_{2}-\frac{\sigma_{2}^{2}}{2}\right) c_{2}+c_{1}^{*}+c_{2}^{*}\right] d t \\
& +e^{t}\left[c_{1} \sigma_{1} d B_{1}(t)+c_{2} \sigma_{2} d B_{2}(t)\right] \\
:= & c^{*} e^{t} d t+e^{t}\left[c_{1} \sigma_{1} d B_{1}(t)+c_{2} \sigma_{2} d B_{2}(t)\right],
\end{aligned}
$$


where $c^{*}=\left(r_{1}-\sigma_{1}^{2} / 2\right) c_{1}+\left(r_{2}-\sigma_{2}^{2} / 2\right) c_{2}+c_{1}^{*}+c_{2}^{*}$. Integrating both sides of it from 0 to $t$, yields

$$
\begin{aligned}
e^{t}\left(c_{1} y_{1}(t)+c_{2} y_{2}(t)\right) & \leq c_{1} y_{1}(0)+c_{2} y_{2}(0)+c^{*}\left(e^{t}-1\right)+\int_{0}^{t} e^{s}\left[c_{1} \sigma_{1} d B_{1}(s)+c_{2} \sigma_{2} d B_{2}(s)\right] \\
c_{1} y_{1}(t)+c_{2} y_{2}(t) & \leq c^{*}+\left(c_{1} y_{1}(0)+c_{2} y_{2}(0)-c^{*}\right) e^{-t}+e^{-t} \int_{0}^{t} e^{s}\left[c_{1} \sigma_{1} d B_{1}(s)+c_{2} \sigma_{2} d B_{2}(s)\right] \\
& \leq c^{*}+c_{1} y_{1}(0)+c_{2} y_{2}(0)+e^{-t} \int_{0}^{t} e^{s}\left[c_{1} \sigma_{1} d B_{1}(s)+c_{2} \sigma_{2} d B_{2}(s)\right] \\
& :=c+e^{-t} \int_{0}^{t} e^{s}\left[c_{1} \sigma_{1} d B_{1}(s)+c_{2} \sigma_{2} d B_{2}(s)\right]
\end{aligned}
$$

where $c=c^{*}+c_{1} y_{1}(0)+c_{2} y_{2}(0)$. It is easy to drive from Lemma 3.7 that

$$
c_{1} y_{1}(t)+c_{2} y_{2}(t) \leq c+O_{\text {a.s. }}(\sqrt{\log t})
$$

which implies

$$
c_{1} \limsup _{t \rightarrow \infty} \frac{y_{1}(t)}{t}+c_{2} \limsup _{t \rightarrow \infty} \frac{y_{2}(t)}{t} \leq 0, \quad \text { a.s. }
$$

Moreover (3.35) together with (3.28) shows that

$$
c_{1} \limsup _{t \rightarrow \infty} \frac{y_{1}(t)}{t} \leq-c_{2} \limsup _{t \rightarrow \infty} \frac{y_{2}(t)}{t} \leq-c_{2} \liminf _{t \rightarrow \infty} \frac{y_{2}(t)}{t} \leq 0, \quad \text { a.s. }
$$

that is,

$$
\limsup _{t \rightarrow \infty} \frac{y_{1}(t)}{t} \leq 0, \quad \text { a.s. }
$$

Similarly, we have

$$
\limsup _{t \rightarrow \infty} \frac{y_{2}(t)}{t} \leq 0, \quad \text { a.s. }
$$

which is as required.

Lemma 3.9. Assume $a_{11}>a_{12}$ and $a_{22}>a_{21}$. Then for any initial value $x_{0} \in R_{+}^{2}$, there exists $a$ positive constant $K(p)$ such that the solution $x(t)$ of system (1.4) has the following property:

$$
E\left[c_{1} x_{1}^{p}(t)+c_{2} x_{2}^{p}(t)\right] \leq K(p), \quad \forall t \in[0, \infty], p>1
$$


Proof. By Itô's formula and Young inequality, we compute

$$
\begin{aligned}
d\left(\frac{1}{p} x_{1}^{p}\right) & =x_{1}^{p-1} d x_{1}+\frac{p-1}{2} x_{1}^{p-2}\left(d x_{1}\right)^{2} \\
& =\left[\left(r_{1}+\frac{p-1}{2} \sigma_{1}^{2}\right) x_{1}^{p}-a_{11} x_{1}^{p+1}+a_{12} x_{1}^{p} x_{2}\right] d t+\sigma_{1} x_{1}^{p} d B_{1}(t) \\
& \leq\left[\left(r_{1}+\frac{p-1}{2} \sigma_{1}^{2}\right) x_{1}^{p}-a_{11} x_{1}^{p+1}+\frac{a_{12} p}{p+1} x_{1}^{p+1}+\frac{a_{12}}{p+1} x_{2}^{p+1}\right] d t+\sigma_{1} x_{1}^{p} d B_{1}(t), \\
d\left(\frac{1}{p} x_{2}^{p}\right) & =x_{2}^{p-1} d x_{2}+\frac{p-1}{2} x_{2}^{p-2}\left(d x_{2}\right)^{2} \\
& =\left[\left(r_{2}+\frac{p-1}{2} \sigma_{2}^{2}\right) x_{2}^{p}+a_{21} x_{1} x_{2}^{p}-a_{22} x_{2}^{p+1}\right] d t+\sigma_{2} x_{2}^{p} d B_{2}(t) \\
& \leq\left[\left(r_{2}+\frac{p-1}{2} \sigma_{2}^{2}\right) x_{2}^{p}+\frac{a_{21}}{p+1} x_{1}^{p+1}+\frac{a_{21} p}{p+1} x_{2}^{p+1}-a_{22} x_{2}^{p+1}\right] d t+\sigma_{2} x_{2}^{p} d B_{2}(t) .
\end{aligned}
$$

Hence, for positive constants $c_{1}$ and $c_{2}$, we have

$$
\begin{aligned}
c_{1} d\left(\frac{1}{p} x_{1}^{p}\right)+c_{2} d\left(\frac{1}{p} x_{2}^{p}\right) \leq & {\left[c_{1}\left(r_{1}+\frac{p-1}{2} \sigma_{1}^{2}\right) x_{1}^{p}-\left(c_{1} a_{11}-\frac{c_{1} a_{12} p}{p+1}-\frac{c_{2} a_{21}}{p+1}\right) x_{1}^{p+1}\right.} \\
& \left.+c_{2}\left(r_{2}+\frac{p-1}{2} \sigma_{2}^{2}\right) x_{2}^{p}-\left(c_{2} a_{22}-\frac{c_{1} a_{12}}{p+1}-\frac{c_{2} a_{21} p}{p+1}\right) x_{2}^{p+1}\right] d t \\
& +c_{1} \sigma_{1} x_{1}^{p} d B_{1}(t)+c_{2} \sigma_{2} x_{2}^{p} d B_{2}(t) .
\end{aligned}
$$

Next, we claim that there are $c_{1}>0, c_{2}>0$ such that

$$
c_{1} a_{11}-\frac{c_{1} a_{12} p}{p+1}-\frac{c_{2} a_{21}}{p+1}>0, \quad c_{2} a_{22}-\frac{c_{1} a_{12}}{p+1}-\frac{c_{2} a_{21} p}{p+1}>0,
$$

if $a_{11}>a_{12}$ and $a_{22}>a_{21}$. In fact, we only need $a_{21} /\left(a_{11}(p+1)-a_{12} p\right)<c_{1} / c_{2}<\left(a_{22}(p+\right.$ 1) $\left.-a_{21} p\right) / a_{12}$, which can be simplified to $a_{12} a_{21}<\left[a_{11}(p+1)-a_{12} p\right]\left[\mathrm{a}_{22}(p+1)-a_{21} p\right]$. It is obviously true, if $a_{11}>a_{12}$ and $a_{22}>a_{21}$. 
Let $\alpha_{1}:=p\left(r_{1}+((p-1) / 2) \sigma_{1}^{2}\right), \alpha_{2}:=p\left(r_{2}+((p-1) / 2) \sigma_{2}^{2}\right), \beta_{1}:=c_{1}^{-(p+1) / p} p\left(c_{1} a_{11}-\right.$ $\left.c_{1} a_{12} p /(p+1)-c_{2} a_{21} /(p+1)\right), \beta_{2}:=c_{2}^{-(p+1) / p} p\left(c_{2} a_{22}-c_{1} a_{12} /(p+1)-c_{2} a_{21} p /(p+1)\right)$. Then $\alpha_{1}>0, \alpha_{2}>0, \beta_{1}>0, \beta_{2}>0$ and

$$
\begin{aligned}
d\left(c_{1} x_{1}^{p}+c_{2} x_{2}^{p}\right) \leq & {\left[c_{1} \alpha_{1} x_{1}^{p}+c_{2} \alpha_{2} x_{2}^{p}-c_{1}^{(p+1) / p} \beta_{1} x_{1}^{p+1}-c_{2}^{(p+1) / p} \beta_{2} x_{2}^{p+1}\right] d t } \\
& +\frac{c_{1}}{p} \sigma_{1} x_{1}^{p} d B_{1}(t)+\frac{c_{2}}{p} \sigma_{2} x_{2}^{p} d B_{2}(t) \\
\leq & {\left[\max \left\{\alpha_{1}, \alpha_{2}\right\}\left(c_{1} x_{1}^{p}+c_{2} x_{2}^{p}\right)-\min \left\{\beta_{1}, \beta_{2}\right\}\left(c_{1}^{(p+1) / p} x_{1}^{p+1}+c_{2}^{(p+1) / p} x_{2}^{p+1}\right)\right] d t } \\
& +\frac{c_{1}}{p} \sigma_{1} x_{1}^{p} d B_{1}(t)+\frac{c_{2}}{p} \sigma_{2} x_{2}^{p} d B_{2}(t) .
\end{aligned}
$$

Hence,

$$
\begin{aligned}
d E\left[c_{1} x_{1}^{p}+c_{2} x_{2}^{p}\right] & \leq\left\{\max \left\{\alpha_{1}, \alpha_{2}\right\} E\left[c_{1} x_{1}^{p}+c_{2} x_{2}^{p}\right]-\min \left\{\beta_{1}, \beta_{2}\right\} E\left[c_{1}^{(p+1) / p} x_{1}^{p+1}+c_{2}^{(p+1) / p} x_{2}^{p+1}\right]\right\} d t \\
& \leq\left\{\max \left\{\alpha_{1}, \alpha_{2}\right\} E\left[c_{1} x_{1}^{p}+c_{2} x_{2}^{p}\right]-2^{-p} \min \left\{\beta_{1}, \beta_{2}\right\} E\left[c_{1} x_{1}^{p}+c_{2} x_{2}^{p}\right]^{(p+1) / p}\right\} d t .
\end{aligned}
$$

By comparison theorem, we can get

$$
\limsup _{t \rightarrow \infty} E\left[c_{1} x_{1}^{p}+c_{2} x_{2}^{p}\right] \leq\left[\frac{2^{p} \max \left\{\alpha_{1}, \alpha_{2}\right\}}{\min \left\{\beta_{1}, \beta_{2}\right\}}\right]^{p}:=C(p),
$$

which implies that there is a $T>0$, such that

$$
E\left[c_{1} x_{1}^{p}+c_{2} x_{2}^{p}\right] \leq 2 C(p), \quad \forall t>T
$$

Besides, note that $E\left[c_{1} x_{1}^{p}+c_{2} x_{2}^{p}\right]$ is continuous, then there is a $\widetilde{C}(p)>0$ such that

$$
E\left[c_{1} x_{1}^{p}+c_{2} x_{2}^{p}\right] \leq \widetilde{C}(p), \quad \forall t \in[0, T] .
$$

Let $K(p)=\max \{2 C(p), \tilde{C}(p)\}$, then

$$
E\left[c_{1} x_{1}^{p}+c_{2} x_{2}^{p}\right] \leq K(p), \quad \forall t \in[0, \infty] .
$$


By the ergodic property, for $m>0$, we have

$$
\lim _{t \rightarrow \infty} \frac{1}{t} \int_{0}^{t}\left(x_{i}^{p}(s) \wedge m\right) d s=\int_{R_{+}^{2}}\left(z_{i}^{p} \wedge m\right) \mu\left(d z_{1}, d z_{2}\right), \quad \text { a.s. }
$$

On the other hand, by dominated convergence theorem, we can get

$$
E\left[\lim _{t \rightarrow \infty} \frac{1}{t} \int_{0}^{t}\left(x_{i}^{p}(s) \wedge m\right) d s\right]=\lim _{t \rightarrow \infty} \frac{1}{t} \int_{0}^{t} E\left[x_{i}^{p}(s) \wedge m\right] d s \leq K(p), \quad i=1,2,
$$

which together with (3.49) implies

$$
\int_{R_{+}^{2}}\left(z_{i}^{p} \wedge m\right) \mu\left(d z_{1}, d z_{2}\right) \leq K(p), \quad i=1,2
$$

Letting $m \rightarrow \infty$, we get

$$
\int_{R_{+}^{2}} z_{i}^{p} \mu\left(d z_{1}, d z_{2}\right) \leq K(p), \quad i=1,2
$$

That is to say, functions $f_{1}(x)=x_{1}^{p}$ and $f_{2}(x)=x_{2}^{p}$ are integrable with respect to the measure $\mu$. Therefore one has the following.

Theorem 3.10. Assume $a_{11}>a_{12}, a_{22}>a_{21}, 2 r_{1}>\sigma_{1}^{2}>0,2 r_{2}>\sigma_{2}^{2}>0$ and $\delta<$ $\min \left\{m_{1}\left(x_{1}^{*}\right)^{2}, m_{2}\left(x_{2}^{*}\right)^{2}\right\}$, where $\delta=\left(a_{21} x_{1}^{*} \sigma_{1}^{2}+a_{12} x_{2}^{*} \sigma_{2}^{2}\right) / 2, m_{1}=a_{11} a_{12}\left(a_{22}-a_{21}\right) / a_{11} a_{22}+a_{12} a_{21}$ and $m_{2}=a_{12}\left(a_{11} a_{22}-a_{12} a_{21}\right) / 2 a_{11}$. Then for any initial value $x_{0} \in R_{+}^{2}$, the solution $x(t)$ of system (1.4) has the following property:

$$
P\left\{\lim _{t \rightarrow \infty} \bar{x}_{1}(t)=\int_{R_{+}^{2}} z_{1} \mu\left(d z_{1}, d z_{2}\right)=\tilde{x}_{1}^{*}\right\}=1, \quad P\left\{\lim _{t \rightarrow \infty} \bar{x}_{2}(t)=\int_{R_{+}^{2}} z_{2} \mu\left(d z_{1}, d z_{2}\right)=\tilde{x}_{2}^{*}\right\}=1 .
$$

Moreover, we can get the following.

Theorem 3.11. Assume $a_{11}>a_{12}, a_{22}>a_{21}$ and $2 r_{1}>\sigma_{1}^{2}, 2 r_{2}>\sigma_{2}^{2}$. Then for any initial value $x_{0} \in R_{+}^{2}$, the solution $x(t)$ has following property

$$
\lim _{t \rightarrow \infty} \frac{1}{t} \int_{0}^{t}\left(a_{11} a_{21} x_{1}^{2}(s)-a_{12} a_{22} x_{2}^{2}(s)\right) d s=a_{21} r_{1} \tilde{x}_{1}^{*}-a_{12} r_{2} \tilde{x}_{2}^{*}
$$

where $\left(\tilde{x}_{1}^{*}, \tilde{x}_{2}^{*}\right)$ is defined as in (3.24). 
Proof. By (3.39), for $\delta>0$, we have

$$
P\left\{\omega: \sup _{(n-1) \delta \leq t \leq n \delta} \frac{x_{1}(t)}{t}>\delta\right\} \leq \frac{E\left[x_{1}^{p}\right]}{(n-1)^{p} \delta} \leq \frac{K(p)}{(n-1)^{p} \delta}, \quad p>1 .
$$

In view of the well-known Borel-Cantelli lemma, we see that for almost all $\omega \in \Omega$,

$$
\sup _{(n-1) \delta \leq t \leq n \delta} \frac{x_{1}(t)}{t} \leq \delta
$$

holds for all but finitely many $n$. Hence there exists a $n_{0}(\omega)$, for all $\omega \in \Omega$ excluding a $P$-null set, for which (3.56) holds whenever $n \geq n_{0}$. Consequently, letting $\delta \rightarrow 0$, we have, for almost all $\omega$

$$
\lim _{t \rightarrow \infty} \frac{x_{1}(t)}{t}=0
$$

Similarly, we can obtain

$$
\lim _{t \rightarrow \infty} \frac{x_{2}(t)}{t}=0
$$

Besides, by (3.39) and its ergodic property, we get

$$
\lim _{t \rightarrow \infty} \frac{1}{t} \int_{0}^{t} x_{1}(s) d B_{1}(s)=0, \quad \lim _{t \rightarrow \infty} \frac{1}{t} \int_{0}^{t} x_{2}(s) d B_{2}(s)=0 .
$$

On the other hand, we have

$$
d\left(a_{21} x_{1}-a_{12} x_{2}\right)=\left[a_{21} x_{1}\left(r_{1}-a_{11} x_{1}\right)-a_{12} x_{2}\left(r_{2}-a_{22} x_{2}\right)\right] d t+a_{21} \sigma_{1} x_{1} d B_{1}(t)-a_{12} \sigma_{2} x_{2} d B_{2}(t) .
$$

Then

$$
\begin{aligned}
a_{21} \frac{x_{1}(t)}{t}-a_{12} \frac{x_{2}(t)}{t}= & a_{21} \frac{x_{1}(0)}{t}-a_{12} \frac{x_{2}(0)}{t}+a_{21} r_{1} \frac{1}{t} \int_{0}^{t} x_{1}(s) d s-a_{12} r_{2} \frac{1}{t} \int_{0}^{t} x_{2}(s) d s \\
& -a_{11} a_{21} \frac{1}{t} \int_{0}^{t} x_{1}^{2}(s) d s+a_{12} a_{22} \frac{1}{t} \int_{0}^{t} x_{2}^{2}(s) d s+a_{21} \sigma_{1} \frac{1}{t} \int_{0}^{t} x_{1}(s) d B_{1}(s) \\
& -a_{12} \sigma_{2} \frac{1}{t} \int_{0}^{t} x_{2}(s) d B_{2}(s) .
\end{aligned}
$$




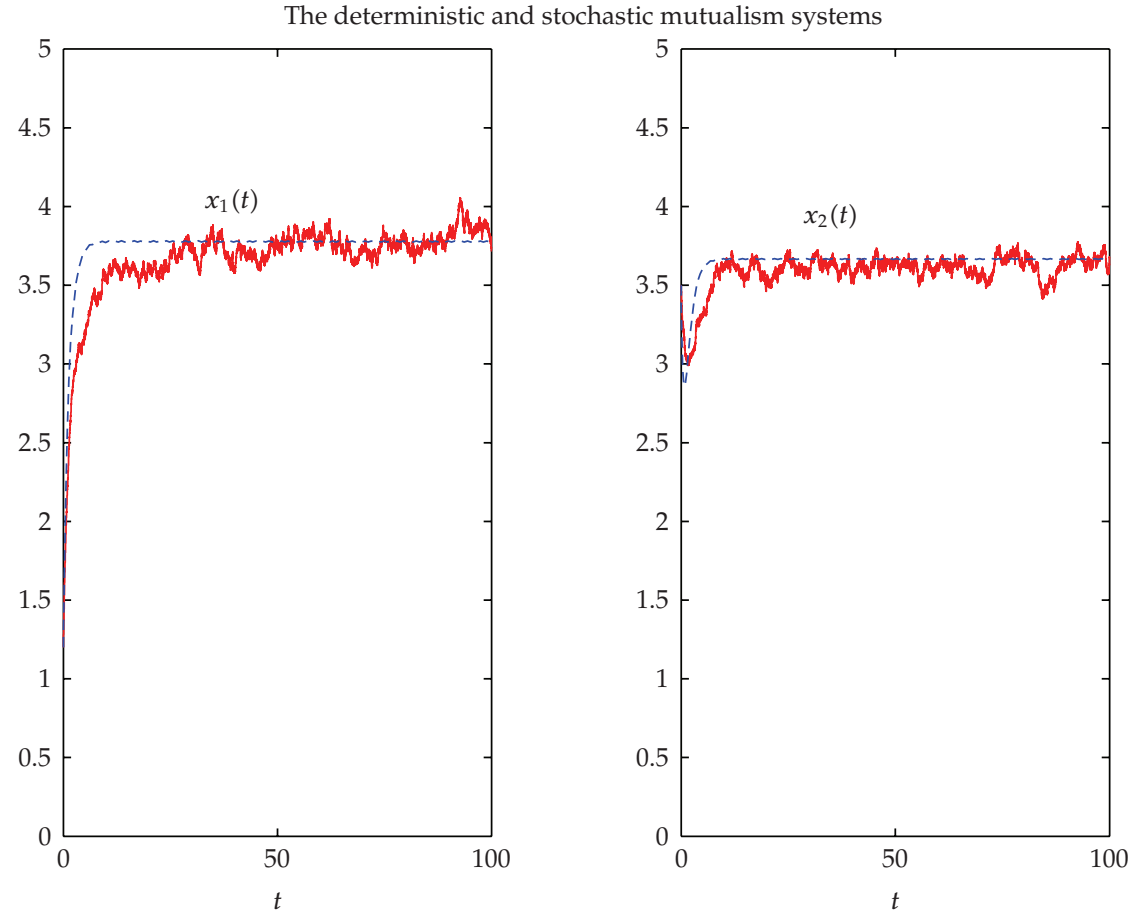

Figure 1: The solution of system (1.1) and system (1.4) with $\left(x_{1}(0), x_{2}(0)\right)=(1.2,3.5), a_{11}=0.6, a_{12}=$ $0.4, a_{21}=0.3, a_{22}=0.5, \sigma_{1}=0.02, \sigma_{2}=0.02$, and $\Delta t=0.001$ such that $a_{11} a_{22}>a_{12} a_{21}$. The imaginary lines represent the solution of system (1.1), and the real lines represent the solution of system (1.4).

Therefore, (3.25), (3.57), (3.58), and (3.59) imply

$$
\lim _{t \rightarrow \infty} \frac{1}{t} \int_{0}^{t}\left(a_{11} a_{21} x_{1}^{2}(s)-a_{12} a_{22} x_{2}^{2}(s)\right) d s=a_{21} r_{1} \tilde{x}_{1}^{*}-a_{12} r_{2} \tilde{x}_{2}^{*}
$$

At the end of this section, to conform the results above, we numerically simulate the solution of (1.4). By the method mentioned in [26], we consider the discretized equation:

$$
\begin{aligned}
& x_{1, k+1}=x_{1, k}+x_{1, k}\left[\left(r_{1}-a_{11} x_{1, k}+a_{12} x_{2, k}\right) \Delta t+\sigma_{1} \epsilon_{1, k} \sqrt{\Delta} t+\frac{1}{2} \sigma_{1}^{2}\left(\epsilon_{1, k}^{2} \Delta t-\Delta t\right)\right], \\
& x_{2, k+1}=x_{2, k}+x_{2, k}\left[\left(r_{2}+a_{21} x_{1, k}-a_{22} x_{2, k}\right) \Delta t+\sigma_{2} \epsilon_{1, k} \sqrt{\Delta} t+\frac{1}{2} \sigma_{1}^{2}\left(\epsilon_{1, k}^{2} \Delta t-\Delta t\right)\right],
\end{aligned}
$$

given the values of $\left(x_{1,0}, x_{2,0}\right)$ and parameters in the system, by Matlab software we get Figure 1 .

Figure 1 gives the solutions of (1.1) and (1.4), and the real lines and the imaginary lines represent the deterministic and the stochastic, respectively. In this figure, we choose parameters such that the conditions said in theorems are satisfied. Hence, although there is no equilibrium of the stochastic system (1.4) as the deterministic system (1.1), but the solution of (1.4) is ergodicity. From the figure, we can see that the solution of system (1.4) is fluctuating around a constant. 


\section{Acknowledgments}

The work was supported by the Ministry of Education of China (no. 109051), the Ph.D. Programs Foundation of Ministry of China (no. 200918), Young Teachers of Northeast Normal University (no. 20090104), and the Fundamental Research Funds for the Central Universities (no. 09SSXT117), the Fundamental Research Funds for the Central Universities.

\section{References}

[1] B. S. Goh, "Stability in models of mutualism," The American Naturalist, vol. 113, no. 2, pp. 261-275, 1979.

[2] J. F. Addicott and H. I. Freedman, "On the structure and stability of mutualistic systems: analysis of predator-prey and competition models as modified by the action of a slow-growing mutualist," Theoretical Population Biology, vol. 26, no. 3, pp. 320-339, 1984.

[3] H. I. Freedman and B. Rai, "Uniform persistence and global stability in models involving mutualism. II. Competitor-competitor-mutualist systems," Indian Journal of Mathematics, vol. 30, no. 2, pp. 175$186,1988$.

[4] X.-Z. He and K. Gopalsamy, "Persistence, attractivity, and delay in facultative mutualism," Journal of Mathematical Analysis and Applications, vol. 215, no. 1, pp. 154-173, 1997.

[5] H. Wu, Y. Xia, and M. Lin, “Existence of positive periodic solution of mutualism system with several delays," Chaos, Solitons and Fractals, vol. 36, no. 2, pp. 487-493, 2008.

[6] Y. Xia, "Existence of positive periodic solutions of mutualism systems with several delays," Advances in Dynamical Systems and Applications, vol. 1, no. 2, pp. 209-217, 2006.

[7] F. Yang and D. Jiang, "Global attractivity of the positive periodic solution of a facultative mutualism system with several delays," Acta Mathematica Scientia A, vol. 22, no. 4, pp. 518-524, 2002.

[8] M. Bandyopadhyay, R. Bhattacharya, and C. G. Chakrabarti, "A nonlinear two-species oscillatory system: bifurcation and stability analysis," International Journal of Mathematics and Mathematical Sciences, no. 31, pp. 1981-1991, 2003.

[9] L. Arnold, W. Horsthemke, and J. W. Stucki, "The influence of external real and white noise on the Lotka-Volterra model," Biometrical Journal, vol. 21, no. 5, pp. 451-471, 1979.

[10] M. Bandyopadhyay and J. Chattopadhyay, "Ratio-dependent predator-prey model: effect of environmental fluctuation and stability," Nonlinearity, vol. 18, no. 2, pp. 913-936, 2005.

[11] M. Carletti, K. Burrage, and P. M. Burrage, "Numerical simulation of stochastic ordinary differential equations in biomathematical modelling," Mathematics and Computers in Simulation, vol. 64, no. 2, pp. 271-277, 2004.

[12] R. Z. Khasminskii and F. C. Klebaner, "Long term behavior of solutions of the Lotka-Volterra system under small random perturbations," The Annals of Applied Probability, vol. 11, no. 3, pp. 952-963, 2001.

[13] E. Renshaw, Modelling Biological Populations in Space and Time, vol. 11 of Cambridge Studies in Mathematical Biology, Cambridge University Press, Cambridge, UK, 1991.

[14] C. Ji, D. Jiang, and N. Shi, "Analysis of a predator-prey model with modified Leslie-Gower and Holling-type II schemes with stochastic perturbation," Journal of Mathematical Analysis and Applications, vol. 359, no. 2, pp. 482-498, 2009.

[15] X. Li and X. R. Mao, "Population dynamical behavior of non-autonomous Lotka-Volterra competitive system with random perturbation," Discrete and Continuous Dynamical Systems A, vol. 24, no. 2, pp. 523-545, 2009.

[16] X. Li, D. Jiang, and X. R. Mao, "Population dynamical behavior of Lotka-Volterra system under regime switching," Journal of Computational and Applied Mathematics, vol. 232, no. 2, pp. 427-448, 2009.

[17] X. R. Mao, G. Marion, and E. Renshaw, "Environmental Brownian noise suppresses explosions in population dynamics," Stochastic Processes and Their Applications, vol. 97, no. 1, pp. 95-110, 2002.

[18] X. R. Mao, S. Sabanis, and E. Renshaw, "Asymptotic behaviour of the stochastic Lotka-Volterra model," Journal of Mathematical Analysis and Applications, vol. 287, no. 1, pp. 141-156, 2003.

[19] C.-H. Zeng, G.-Q. Zhang, and X.-F. Zhou, "Dynamical properties of a mutualism system in the presence of noise and time delay," Brazilian Journal of Physics, vol. 39, no. 2, pp. 256-259, 2009.

[20] L. Arnold, Stochastic Differential Equations: Theory and Applications, John Wiley \& Sons, New York, NY, USA, 1972.

[21] X. R. Mao, Stochastic Differential Equations and Applications, Horwood, Chichester, UK, 1997. 
[22] D. Jiang and N. Shi, "A note on nonautonomous logistic equation with random perturbation," Journal of Mathematical Analysis and Applications, vol. 303, no. 1, pp. 164-172, 2005.

[23] I. N. Wantanabe, Stochastic Differential Equations and Diffusion Processes, North-Holland, Amsterdam, The Netherlands, 1981.

[24] T. Caraballo and P. E. Kloeden, "The persistence of synchronization under environmental noise," Proceedings of the Royal Society of London A, vol. 461, no. 2059, pp. 2257-2267, 2005.

[25] R. Z. Hasminskii, Stochastic Stability of Differential Equations, vol. 7 of Monographs and Textbooks on Mechanics of Solids and Fluids: Mechanics and Analysis, Sijthoff \& Noordhoff, Alphen aan den Rijn, The Netherlands, 1980.

[26] D. J. Higham, "An algorithmic introduction to numerical simulation of stochastic differential equations," SIAM Review, vol. 43, no. 3, pp. 525-546, 2001. 


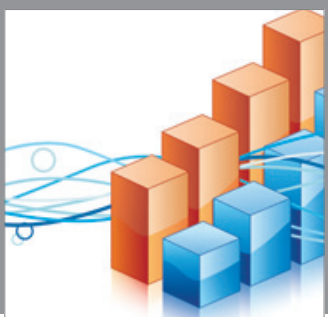

Advances in

Operations Research

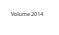

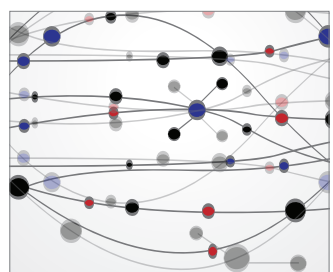

\section{The Scientific} World Journal
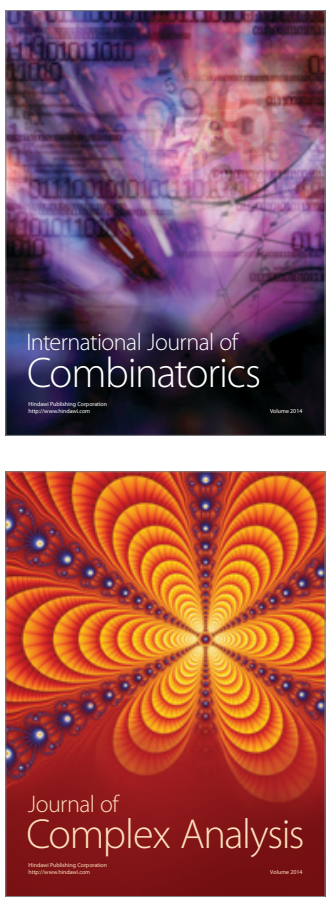

International Journal of

Mathematics and

Mathematical

Sciences
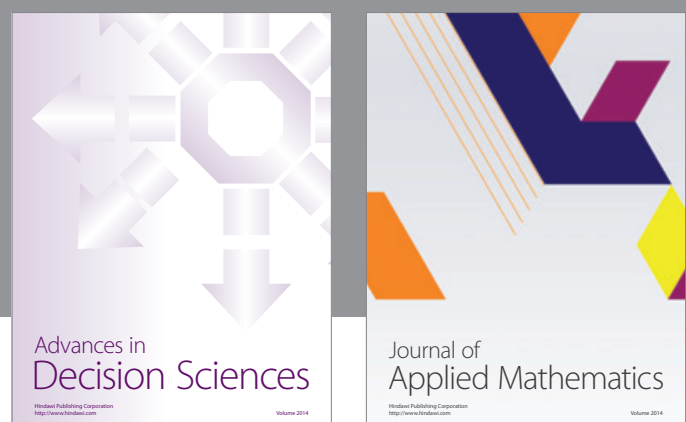

Journal of

Applied Mathematics
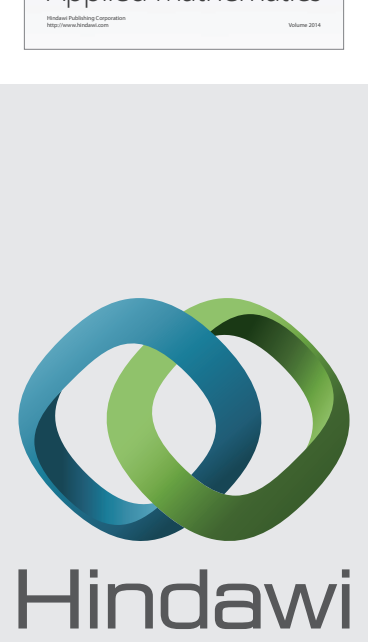

Submit your manuscripts at http://www.hindawi.com
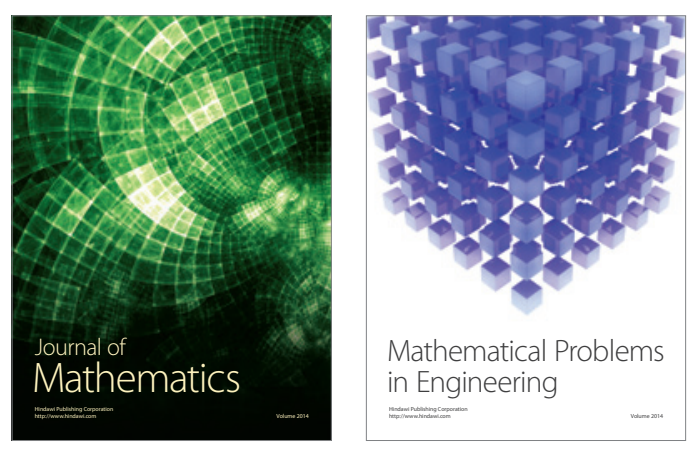

Mathematical Problems in Engineering
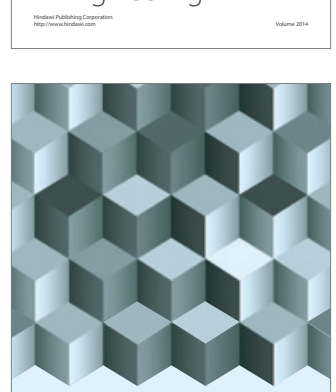

Journal of

Function Spaces
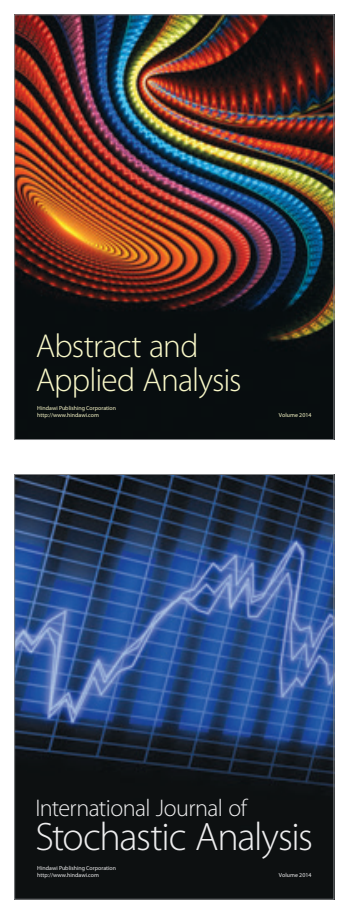

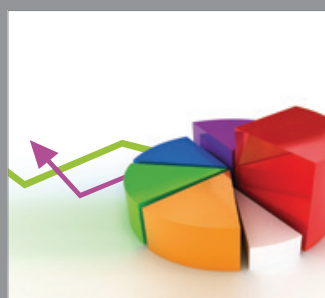

ournal of

Probability and Statistics

Promensencen
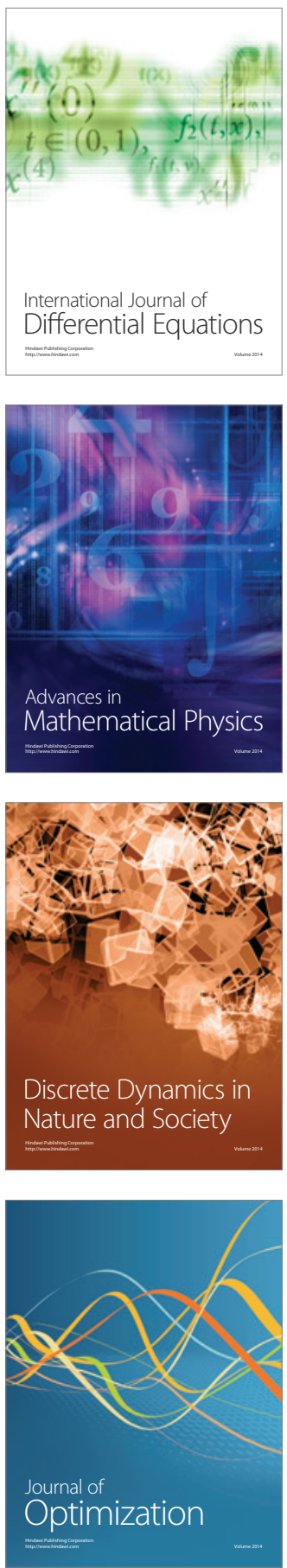